\title{
Evaluation of Mechanical Properties of MEMS Materials and Their Standardization
}

Toshiyuki Tsuchiya, Department of Micro Engineering, Kyoto University, Japan

\begin{abstract}
The importance of the mechanical properties evaluation on designing and evaluating MEMS and the development of standard on MEMS are described in this chapter. First, in order to confirm their importance, the effect of mechanical properties on the performance of MEMS is pointed out. Second, to reveal the accuracy and repeatability of the existing evaluation methods, a work for cross comparisons is described. Then, the current workings on the international standard development on thin film mechanical properaties to improve the reliability, repeatability, and accuracy in the mechanical properties evaluation are introduced.
\end{abstract}

\section{Keywords}

thin films; mechanical properties; MEMS; tensile testing; standardization

\subsection{Introduction 2}

1.2 Thin-film Mechanical Properties and MEMS 3

1.2.1 Elastic Properties 4

1.2.2 Internal Stress 5

1.2.3 Strength 6

1.2.4 Fatigue 7

1.3 Issues on Mechanical Properties Evaluations 8

1.3.1 Issues Related to Specimens 8

1.3.2 Issues Related to Test Apparatus 8

1.3.3 Standards 10

1.4 Cross-comparison of Thin-film Tensile Testing Methods 10

1.4.1 Tensile Testing Methods 11

1.4.2 Specimen Design 12

1.4.3 Materials 13

1.4.4 Specimen Fabrication 14

1.4.5 Results 15

1.4.5.1 Single-crystal Silicon and Polysilicon 16 


$\begin{array}{ll}\text { 1.4.5.2 } & \text { Nickel 18 } \\ \text { 1.4.5.3 } & \text { Titanium 19 } \\ \text { 1.4.6 } & \text { Discussion } 21\end{array}$

1.5 International Standards on MEMS Materials 22

1.5.1 MEMS Standardization Activities 22

1.5.1.1 IEC 22

1.5.1.2 ASTM International 22

1.5.1.3 SEMI 23

1.5.1.4 Micromachine Center in Japan 23

1.5.2 International Standards on Thin-film Uniaxial Stress Testing 23

1.6 Conclusion 24

References 24

1.1

Introduction

Evaluations of the mechanical properties of micro- and nano-materials, especially thin films, which form mechanical structures of microelectromechanical system (MEMS) devices, are significant irrespective of the commercialization of applied devices for MEMS. The properties of thin films have been evaluated to satisfy demands in semiconductor device research, but they were mainly on the electrical properties. Studies on evaluations of mechanical properties have been limited, mainly to internal stresses. When the mechanical properties were needed, the bulk properties were often adopted, which was sufficient for their demands. However, when thin films started to be used for various mechanical structures, the mechanical and electromechanical properties play important roles in the operation of MEMS devices. Therefore, the mechanical properties of thin films need to be measured, and accurate properties similar to the electrical properties in semiconductor devices are required.

The mechanical properties of thin films should be measured on the same scale as micro- and nano-devices, since they are different from those of bulk materials. Reasons of the differences are follows;

- Size effects: The ratio of the surface area to the volume increases with decrease in the dimensions of a device structure. The surface effect might be more effective in MEMS devices. For example, the fracture of silicon, a brittle material, was initiated from the surface defects that are mainly produced during the fabrication process and the surface roughness dominates the strength. The size effect would be more sensitive at the microscale.

- Thin-film materials: Thin-film materials often have different compositions, phase and microstructure from the bulk materials, even if they are called by the same material names. The formation processes, such as deposition, 
thermal treatment, implantation and oxidation, are inherent methods for thin-film materials. For example, bulk "silicon nitride" is a polycrystalline material and often contains impurities for improving properties, but silicon nitride thin films are deposited by chemical vapor deposition and are amorphous and seldom doped by impurities.

- Processing: Mechanical processing, which is the most commonly used processing method for bulk structure, is rarely used because the processing speed is too fast for the microscale. Instead, photolithography and etching are widely used. The surface finishing of the processed structure is completely different between the bulk and thin film.

These are the reasons for the necessity for the direct measurement of thin-film materials. In addition, it reveals the effects of their formation, processing and dimensions on their mechanical properties. The dimensions of the structures in MEMS devices have wide ranges, from sub-micrometers to millimeters. Evaluations of the mechanical properties of thin films cover a very wide range of measurement scale. Many measurement methods have been developed and various values have been measured using these methods.

However, studies on both the development of measurement methods and the evaluation of thin-film materials showed that there were inaccuracies in the measured results obtained by each method. The variations in the measured properties were large but the source of the variation was not established since there were too many differences among the properties measured by the different methods. The accuracy of the measurement methods, which is the basis of the evaluation, has not been verified because there are no standards for the mechanical properties of thin films. Recently, the development of international standards for measurements of mechanical properties was initiated in order to obtain more accurate properties and reliable measurements.

In this chapter, the importance of the mechanical properties for MEMS devices is defined to confirm the necessity for the evaluation of method developments and their standardization. The effects of each mechanical property on the design and evaluation of the devices are pointed out. Then, cross-comparisons of the evaluation methods for mechanical properties are described to indicate the critical points for more accurate measurements at the thin-film scale. Finally, current progress in the development of international standards on thin-film mechanical properties to improve the reliability, repeatability and accuracy of measurements of mechanical properties is discussed.

\section{2}

\section{Thin-film Mechanical Properties and MEMS}

The evaluation of the mechanical properties of thin films is indispensable for designing MEMS devices, since the properties play the following roles: 
- Device performance: In MEMS devices, the mechanical properties are closely connected to the device performance. Accurate values of the mechanical properties are needed for obtaining the best performance.

- Reliability: MEMS devices are intended to be used in harsh environments because of their small size. Reliability is one of the most important properties.

In addition, the establishment of a properties database is required in order to accumulate knowledge about design information. Recently, the rapid prototyping of MEMS devices by incorporating MEMS foundry services and CAD/CAE software dedicated to MEMS devices has attracted much interest. A suitable database of thin-film mechanical properties should be compiled in order to ensure the most appropriate designs. This section provides descriptions of the effect of each mechanical property on the properties of MEMS devices to emphasize the importance of their evaluation.

\subsection{1}

\section{Elastic Properties}

Elastic properties, such as Young's modulus, Poisson's ratio and shear modulus, are directly related to the device performance. The stiffness of a device structure is proportional to the Young's modulus or shear modulus and the resonant frequency is proportional to the square root. However, as discussed in the next section, the stiffness of the thin-film structure depends additionally on the internal stress and the internal stress changes by an order of the magnitude, and these effects of the elastic properties on the device performance should be considered as a maximum effect. The acceptable errors in the elastic properties will be a few percent for cantilever beam structures and folded beam structures in which the internal stress has no effect on the stiffness of the structure. Larger deviations will be acceptable for structures whose internal stress dominates their stiffness. The stiffness of membrane structures for pressure sensors and diaphragm pumps is affected by the Poisson's ratio. The pressure $P$ and center deflection $w_{0}$ of a circular membrane, as shown in Figure 1.1(a), are expressed by

$$
P=\frac{4 \sigma_{0} h}{r^{2}} w_{0}+\frac{8 E h}{3(1-v) r^{4}} w_{0}^{3}
$$

where $h, r, E, \sigma_{0}$ and $v$ are the thickness, radius, Young's modulus, internal stress and Poisson's ratio of the membrane, respectively. The range of the Poisson's ratio of materials is not wide and the effect is not large, as shown in Eq. (1). A rough estimation of the Poisson's ratio by using the bulk properties is often acceptable.

These arguments will lead to the conclusion that the temperature coefficients of the elastic properties are negligible for most sensor devices. For a specific application, such as oscillators and filters which use MEMS structure as resona- 
tors, the deviation and the temperature coefficient should be more precisely measured and controlled. They require stability of the resonant frequency of the order of ppm.

\section{2 .2}

\section{Internal Stress}

The internal stress, the strain generated in thin films on thick substrates, is not an elastic property in the strict sense. If the stress is present along the longitudinal direction for a doubly supported beam structure and the in-plane direction for a fixed-edge membrane structure, the stiffness along the out-of-plane direction of the structure has terms of the internal stress. Since the internal stress has an effect similar to large displacement analysis, the effect of internal stress on the stiffness and resonant frequency should be considered as closely as that of the Young's modulus.

The doubly supported beams shown in the Figure 1.1(b), and also the membranes shown in Figure 1.1(a), are loaded by the internal stress, hence the stiffness will change. The lateral stiffness of the doubly supported beam structure shown in Figure 1.1(b) is described by

$$
F=\frac{4 E b h^{3}}{l^{3}}\left[1+\frac{\pi^{2} \sigma_{0}}{8 E}\left(\frac{l}{h}\right)^{2}+\frac{\pi^{4}}{128}\left(\frac{w_{0}}{h}\right)^{2}\right] w_{0}
$$
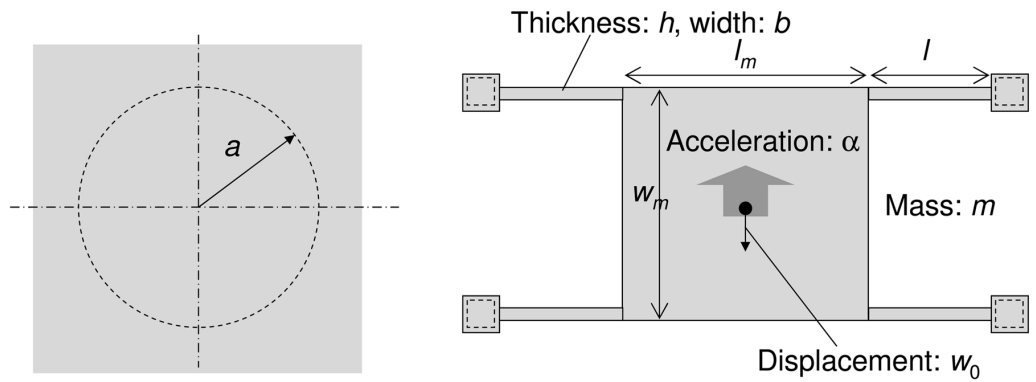

Thickness: $h \quad$ Pressure: $p$

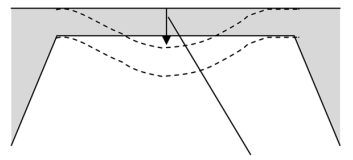

Displacement at center: $w_{0}$

(a)

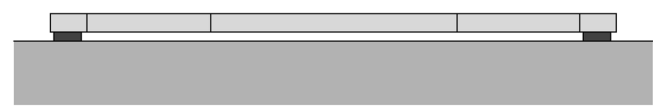

(b)

AMNS-6 MEMS Reliability

Figure 1.1 Typical structures of MEMS devices. (a) Thin diaphragm for pressure sensors; (b) doubly supported massbeam structure for resonator and accelerometer. 
where $l$ and w are the length and width, respectively, of the beams. If the center mass consists of thin films and the stress in the mass is released, the additional stress is applied on the supporting beam. In this case, the modified stress is described by

$$
\sigma_{\mathrm{c}}=\sigma_{0}\left(1+\frac{l_{\mathrm{m}}}{2 l}\right)
$$

where the $l_{\mathrm{m}}$ is the mass length.

The range of the internal stress values is wide; in the case of polysilicon, the stress range is from -500 to $700 \mathrm{MPa}$ depending on the deposition methods and conditions and heat treatments. The negative (compressive) stress causes a decrease in the stiffness. Zero stiffness leads to the buckling of the structure. Stress control and accurate measurement are more important factors.

The origin of internal stress is classified into the intrinsic stress and the thermal stress. Chemical reactions, ion bombardment, absorption and adsorption cause the intrinsic stress, which can be controlled by the deposition conditions. However, control of the repeatability of the process conditions and resulting internal stress is very difficult. The thermal stress is caused by the mismatch of the coefficients of thermal expansion of the thin film and of its substrate. The thermal stress often becomes the origin of the temperature properties of the structures; the release or control of the thermal stress should be considered in designing device structures.

The internal stress may cause the destruction of the structure. High compressive stress causes buckling as discussed above and high tensile stress causes the fracture of structures. In both cases, film peeling is possible with large stresses.

The internal stress considered above is assumed to be uniform along the thickness direction. Actually, the stress is often distributed along the thickness direction, which causes out-of-plane deflection of cantilever structures.

\section{2 .3}

\section{Strength}

The strength of the thin-film materials need to be evaluated and controlled to assure and improve the reliability of MEMS devices. The strength is the main parameter for the deposition process, etching, microstructures and shape uniformity. These parameters should be considered in order to evaluate the reliability. When engineers apply the measured strength values to their own devices, they should consider not only the test methods but also the fabrication methods of the specimens. For example, on designing the strength of a membrane structure which is to be used for a pressure sensor and has no etched surfaces, the tensile or bending strength of cantilever beam specimens should never be used because the beam structure has etched surfaces, dominating the fracture properties. In addition, the loading direction should be considered when evaluating beam structures. The lateral and vertical strengths may be different even if the same specimen is tested. 
MEMS devices are expected to be used in mobile and portable applications, where the system and device structures are expected to have high durability against shocks. The requirement for shock durability often causes the difficult device design because the stress generated by the shock is larger than the stress applied in their normal operation. For example, accelerometers for automobile and mobile applications are designed to have measurement ranges of few to few tens of $G$ (gravity). However, the shock applied with a drop from a height of $1.5 \mathrm{~m}$ on to a concrete surface is said to be equivalent to 3000-10000 G. If the shock is applied directly, the device structure will have a stress of at least 100 times larger than the stress due to the designated input of the sensor. Therefore, the device has a stopper as a shock reduction structure. When there is no stopper structure because of the fabrication capability, the device sensitivity is limited to reducing the stress during shock.

In the case of vibrating gyroscopes that measure the Coriolis force to sense the angular rate, the shock can be reduced by adding a damper in the packaging structures. Accelerometers do not have such a damper because it causes a reduction in response time.

\subsection{4}

\section{Fatigue}

Fatigue is observed as a change in elastic constants, plastic deformation and strength decrease through the application of a cyclic or constant stress for a long time. Plastic deformation and changes in elastic constants cause sensitivity changes and offset drift in devices and fatigue fracture causes sudden failure of the device functions. These should be avoided in order to realize highly reliable devices.

Silicon, the most widely used structural material, shows no plastic deformation at room temperature. In addition, silicon was thought to show no fatigue fracture, which means that it suffers no decrease in strength on long term application of stress. Therefore, previously some engineers did not consider the fatigue of silicon MEMS devices. However, various experiments have shown fatigue fracture and decrease in strength of more than few tens of percent of the initial strength. Now all MEMS engineers consider the reliability of silicon structures to increase the device reliability.

Metal films, such as aluminum and gold, which are used in micromirror devices, show plastic deformation and metallic structures may show large drift and changes in performances. For example, digital micro mirror devices (DMDs) [1] are operated by on-off state, which is acceptable for change in material properties.

As for the size effect of MEMS structures, the surface effect will contribute greatly to the fatigue properties. The effect of the environment, such as temperature and humidity, should be evaluated. The resonant frequencies of the MEMS structures are higher than those of macroscale devices. In order to assure the long-term reliability of such devices, we should evaluate the reliability against a large number of cyclic stress applications. If we assume that the resonant frequency of the device is about $10 \mathrm{kHz}, 1$ year of continuous operation equals $10^{11-12}$ 
cycle loadings. Therefore, proper accelerated life test method and life prediction method are required by analyzing the mechanism of the fatigue behavior.

\section{3}

\section{Issues on Mechanical Properties Evaluations}

In the design of MEMS devices and confirmation of their reliability, evaluations of the mechanical properties of micro- and nano-materials are crucial. However, there are some issues regarding accurate measurements, which are related mainly to the accuracy of the measured values.

\subsection{1}

\section{Issues Related to Specimens}

Deviations of specimen dimensions are one of the most important and basic issues in evaluating the properties of thin films. In bulk mechanical structures, the dimensions of structures are made highly accurate by means of machining tools and measurement tools. A mechanically machined structure can be made with a precision of more than one thousandth of its dimensions. However, in MEMS structures, although the absolute error in fabrication is smaller than in mechanically machined structures, the relative accuracies are not good, because the total dimensions are much smaller than the errors. Regarding the thickness of the structure, the deviation is a few percent for most of the deposition methods. In addition, silicon on insulator wafers, whose device layer thickness is determined by the polishing process, has relatively large deviations in the thickness if the device layer is as thin as a few micrometers, because the uniformity of the polishing process is about $0.5-1 \mu \mathrm{m}$, irrespective of the total thickness. The lateral dimension, which is mostly determined by photolithography and etching, has the same order of deviation.

Not only the deviation but also the variation of the dimensions of the structure becomes an issue regarding measurement accuracy. Figure 1.2 shows the dimensions of specimens used in published papers on tensile tests of both single-crystal silicon and polysilicon thin films [2-12]. The horizontal and vertical axes represent the length and cross-sectional area of the specimen parallel part, respectively. The plot shows clearly the difference in the dimensions of the thin film specimens. Since the size effect should be considered, direct comparison between the tensile strengths of these specimens is difficult because the dimensions were varied over wide ranges.

\subsection{2}

\section{Issues Related to Test Apparatus}

The differences in the measurement methods become another issue. It is difficult to attribute the differences in measured mechanical properties between measure- 


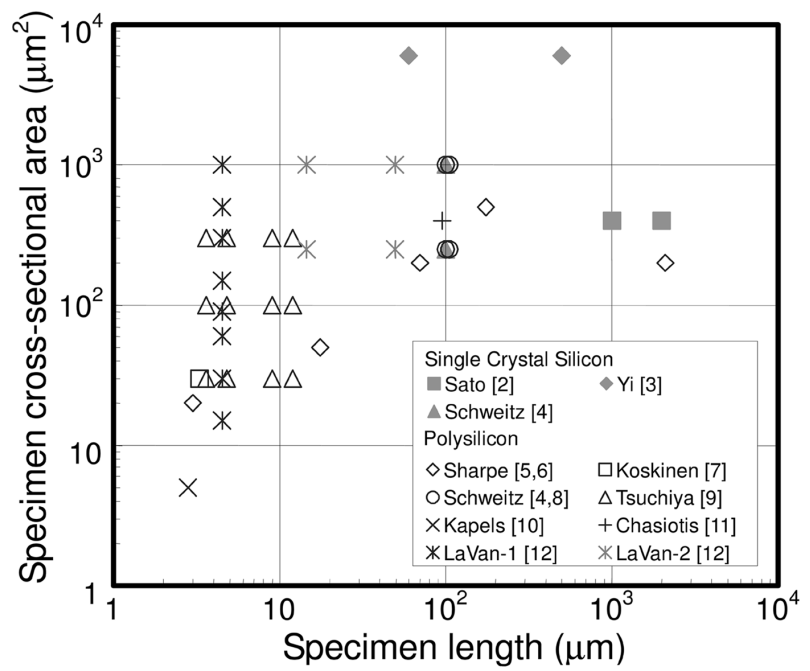

Figure 1.2 Dimensions of previously reported thin-film specimen for uniaxial tensile test. LaVan's specimens [12] are categorized into two types.

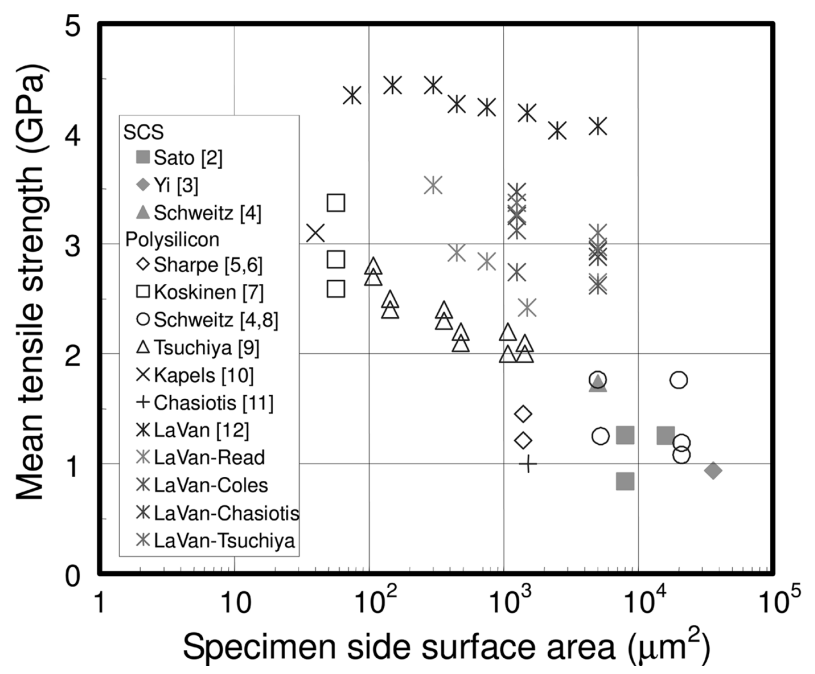

Figure 1.3 Reported tensile strength of both single-crystal silicon and polysilicon films. LaVan's specimens [12] were tested by five institutes.

ment methods. They may include the deviations of all possible parameters, as discussed above. Figure 1.3 shows the measured tensile strength of silicon specimens in the same paper as in Figure 1.2. The average tensile strength was plotted against the side-surface area, which is twice the product of the length and thickness of the specimen parallel part. The plot is based on the result of the size effect 
analysis of polysilicon specimens, which shows that the fracture origin is located on the side surfaces that are processed by reactive ion etching processes. Brittle materials, whose fracture is dominated by defects contained in the specimen, exhibit a size effect on the strength. The fracture in silicon thin films was often initiated from the side surface of specimens that were formed by dry etching [9]. Therefore, the size effect on strength should be normalized by the side-surface area of the specimens. As can be seen in Figure 1.3, the effect of specimen size appeared in the same experiments and the slope of strength against side-surface area was similar for all experiments. However, the size effect between the different experiments was not clearly observed.

\subsection{3}

\section{Standards}

In methods to evaluate the material properties of bulk materials, international standards are usually established to minimize differences and errors between test machines in measuring properties. Standards on test machines, test specimens and standard specimens to calibrate test machines were established and used to improve the reliability and accuracy of test results. However, standards on the mechanical properties of thin films have not been investigated or established.

The lack of standard methods on the evaluation of thin-film mechanical properties prevents effective material research, as discussed above. It was concluded that many reasons are responsible for the differences between measurements, such as deposition conditions, post-annealing, etching, specimen size effect, deviations in dimensions of specimens, stress concentrations caused by specimen shape and errors resulting from the test apparatus. However, the source of these differences has not been attributed quantitatively and the reliability of each measured value is not confirmed because standard procedures and methods for thin films have not been established.

\section{4}

\section{Cross-comparison of Thin-film Tensile Testing Methods}

To investigate whether differences in tensile strength were caused by the test method, a cross-comparison of existing tensile test methods was carried out. It is difficult to compare test methods from the reported results, as discussed the previous section. Specimens made of the same materials fabricated with the same processes have to be tested in parallel. A round robin test (RRT) scheme was applied to compare the test methods to eliminate the effects of materials, processes, specimen shapes and dimensions. RRTs are evaluations conducted on one specimen at different locations or with different methods to compare the results so that each test method can be checked and evaluated. However, in this case, it is not possible to evaluate the same specimen in the strict sense, since specimens were broken during the fracturing tests. Therefore, the same specimen in this RRT 


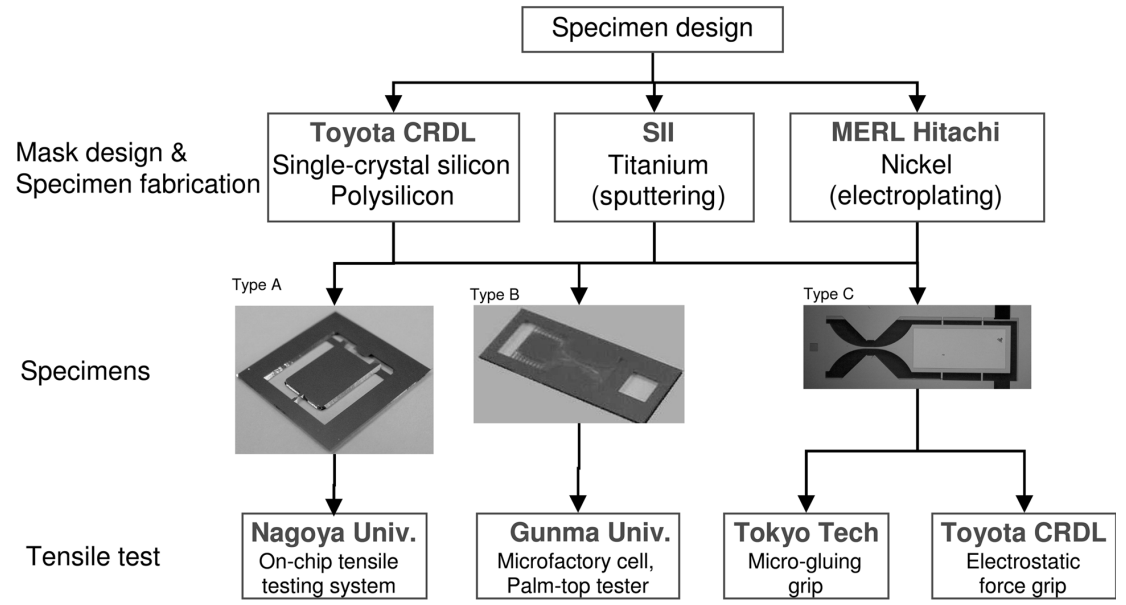

Figure 1.4 Plan to implement round robin test.

was defined as samples fabricated by the same process on a single wafer. Since it is possible to produce multiple microstructures simultaneously with a batch process using silicon micromachining, we can produce samples that are practically the same. We can also minimize variations in the specimen manufacturing process by producing the specimens simultaneously on a single wafer, because the test material undergoes the same fabrication process.

The plan to implement the round robin tests is shown in Figure 1.4. Specimens were designed based on three types of shape conforming to the test methods that will be described below. Three institutes conducted the mask design and fabrication process for each test material. Specimens extracted from a single wafer were distributed to four institutes that conducted tests on these according to the institute's methods.

\subsection{1}

\section{Tensile Testing Methods}

Table 1.1 lists the tensile test methods that were compared in the RRTs. These were characterized by their specimen gripping methods. Tensile stress loading was done by piezoelectric actuators or motorized micrometers. The tensile load was measured with a load cell or the displacement of a double cantilever beam. Elongation in the specimen was measured by gauge mark displacement using image analysis.

Sato et al. at Nagoya University employed an on-chip tensile testing system [2] that integrates a tensile-stress loading system with the specimen chip. The chip converts vertical external load to tensile force on the specimen. From the vertical load and the displacement of the load lever, one can calculate the stress and strain 
12 Evaluation of Mechanical Properties of MEMS Materials and Their Standardization

Table 1.1 Tensile test methods compared in the round robin test.

\begin{tabular}{|c|c|c|c|c|c|c|}
\hline Institute & Method & Tensile loading & $\begin{array}{l}\text { Load (stress) } \\
\text { measurement }\end{array}$ & $\begin{array}{l}\text { Strain } \\
\text { measurement }\end{array}$ & Specimen & Ref. \\
\hline $\begin{array}{l}\text { Nagoya } \\
\text { University } \\
(\mathrm{NU})\end{array}$ & $\begin{array}{l}\text { On-chip } \\
\text { tensile } \\
\text { testing } \\
\text { system }\end{array}$ & $\begin{array}{l}\text { Motorized } \\
\text { micrometer }\end{array}$ & $\begin{array}{l}\text { Double } \\
\text { cantilever } \\
\text { beam }\end{array}$ & $\begin{array}{l}\text { Image } \\
\text { analysis }\end{array}$ & Type A & 2 \\
\hline $\begin{array}{l}\text { Gunma } \\
\text { University } \\
(\mathrm{GU})\end{array}$ & $\begin{array}{l}\text { Microfactory } \\
\text { cell, palm- } \\
\text { top tester }\end{array}$ & $\begin{array}{l}\text { Piezo-driven } \\
\text { inch-worm }\end{array}$ & $\begin{array}{l}\text { Double } \\
\text { cantilever } \\
\text { beam }\end{array}$ & $\begin{array}{l}\text { Image } \\
\text { analysis }\end{array}$ & Type B & 13 \\
\hline $\begin{array}{c}\text { Toyota CRDL } \\
\text { (TCRDL) }\end{array}$ & $\begin{array}{l}\text { Electrostatic } \\
\text { grip }\end{array}$ & Piezo-actuator & Load cell & $\begin{array}{l}\text { Image } \\
\text { analysis }\end{array}$ & Type C & 9 \\
\hline Tokyo Tech & $\begin{array}{l}\text { Micro-gluing } \\
\text { grip }\end{array}$ & $\begin{array}{l}\text { Magnetostrictive } \\
\text { actuator }\end{array}$ & Load cell & $\begin{array}{l}\text { Image } \\
\text { analysis }\end{array}$ & Type C & 14 \\
\hline
\end{tabular}

on the specimen by differentiating two measurements of the load-deflection relationship before and after the specimen's fracture.

Saotome et al. at Gunma University [13] used mechanical grip systems applied to thin-film specimens. A cantilever-shaped thin-film specimen was fabricated on a silicon wafer and the free end was fixed to the silicon frames by support beams. After being placed on the tester and fixed by the grip, the specimen was released from the frame by breaking the support beams.

A micro-gluing grip was employed by Higo et al. [14] at Tokyo Institute of Technology (Tokyo Tech), which uses an instant glue to fix the micro-sized specimen. An electrostatic grip was employed by Tsuchiya et al. at Toyota Central Research and Development Laboratories (TCRDL) [9], which uses electrostatic force to chuck the specimen. A cantilever beam with large paddles on its free end was used as the specimen. Electrostatic force for fixing the specimen was generated by applying voltage between the specimen and the chuck device (probe) for conductive materials.

\section{4 .2}

\section{Specimen Design}

Three types of RRT specimens were designed. We would have preferred to test specimens with the same shape for the RRTs. However, the specimen shapes and dimensions are completely different from one another because of the test methods. In the five test methods, the size of the specimen chip of the on-chip testing device is $15 \mathrm{~mm}$ square, whereas that of the electrostatic force grip is only $1 \mathrm{~mm}$ square. It is impossible to use one specimen design for all test methods. Therefore, we used three different designs that had the same length and width over the gauge (parallel) part. We determined the specimen design and dimensions taking the specimen dimensions in Figure 1.1 into consideration. However, the length of the 


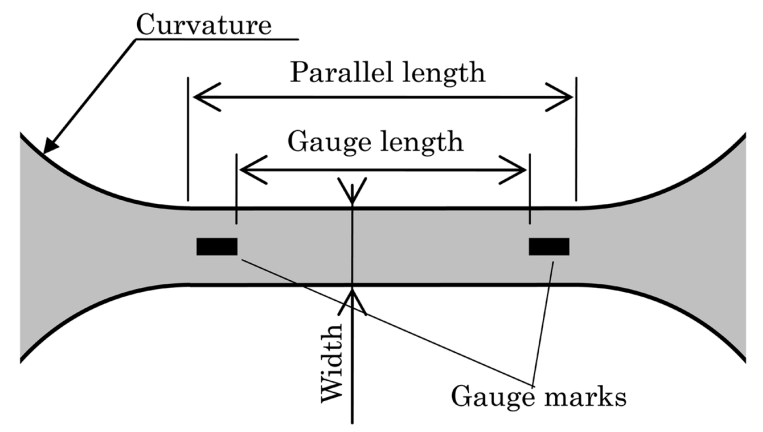

Figure 1.5 Tensile testing part of round robin specimen.

Table 1.2 Dimensions of the specimens for each test method.

\begin{tabular}{|c|c|c|c|c|}
\hline Specimen type & $A$ & $B$ & \multicolumn{2}{|l|}{$C$} \\
\hline Test method & On-chip & Mechanical & \multicolumn{2}{|c|}{ Glue/electrostatic } \\
\hline Width $(\mu \mathrm{m})$ & 50 & 50 & 50 & 50 \\
\hline Gauge length ( $\mu \mathrm{m})$ & 100 & 500 & 100 & 500 \\
\hline Parallel length ( $\mu \mathrm{m})$ & 120 & 600 & 120 & 600 \\
\hline Curvature ( $\mu \mathrm{m})$ & 500 & 5000 & 500 & 500 \\
\hline No. of specimens & 13 & 14 & 24 & 24 \\
\hline
\end{tabular}

three types for specimens could not be designed to be the same. The maximum specimen length for the on-chip test was $100 \mu \mathrm{m}$ and the minimum length of the mechanical grip was $500 \mu \mathrm{m}$. Therefore, the electrostatic/gluing specimen had two types of specimens of different lengths to make comparisons among all methods.

The design of the testing part is shown in Figure 1.5 and the dimensions of each specimen type and the number on one wafer are summarized in Table 1.2. We placed as many of the three types of RRT specimens as possible on a 4-inch wafer, because all the RRT specimens had to be obtained from the one wafer fabricated through the same process.

\section{4 .3}

\section{Materials}

Single-crystal silicon, polysilicon, nickel and titanium thin films were selected as the test materials considering their application to micromachines as structural materials. The deposition method and thickness of the test materials are summarized in Table 1.3. 
Table 1.3 Thin-film materials evaluated in the round robin test.

\begin{tabular}{|c|c|c|c|}
\hline Fabrication & Material & Method & $\mathrm{t}(\mu \mathrm{m})$ \\
\hline \multirow[t]{3}{*}{ TCRDL } & SCS SDB & $\mathrm{CZ} \rightarrow$ direct bond & 2.0 \\
\hline & Epi & Epitaxial & 2.0 \\
\hline & Polysilicon & LPCVD & 2.0 \\
\hline Hitachi & Nickel & Electroplating & 2.0 \\
\hline SII & Titanium & Sputtering & $0.5,1.0$ \\
\hline
\end{tabular}

Silicon is the most frequently used material for micromachine structures because of its superior elastic properties. A single-crystal silicon (SCS) specimen was fabricated from the top layer of a silicon-on-insulator (SOI) wafer. There are various fabrication methods for an SOI wafer: silicon direct bonding (SDB) SOI (SDB-SOI), oxygen ion implantation (SiMOX) and epitaxial growth of silicon \{EpiSOI(CANON ELTRAN [15])). We used SDB-SOI and Epi-SOI because the former is commonly used and the latter has better thickness uniformity $(\sim 3 \%)$ than SDB-SOI $( \pm 0.5 \mu \mathrm{m})$. In this chapter, we call these SCS specimens fabricated from SDB-SOI and Epi-SOI SCS/SDB and SCS/Epi, respectively. For the polysilicon specimen, we used a crystallized film from low-pressure chemical vapor-deposited (LPCVD) amorphous silicon using disilane $\left(\mathrm{Si}_{2} \mathrm{H}_{6}\right)$ gas as source gas [16].

Electroplated nickel film is also used in LIGA (Lithographie, Galvanoformung, Abformung) processes and other electroplated structures. The test materials deposited on a wafer need to be low tensile stress films and have small thickness variations $(<5 \%)$. For a nickel specimen, nickel(II) sulfamate tetrahydrate $\left[\mathrm{Ni}\left(\mathrm{OSO}_{2} \mathrm{NH}_{2}\right)_{2} \cdot 4 \mathrm{H}_{2} \mathrm{O}\right]$ solution was used as the electrolyte and the current density and the temperature of electroplating were $0.51 \mathrm{Adm}^{-2}$ and $51^{\circ} \mathrm{C}$, respectively, which were optimized for thickness uniformity and internal stress control.

We also selected sputtered titanium films, which are widely used as an electrode material. The titanium film was deposited by sputtering. The argon gas flow rate was controlled and an intermediate cooling process was used for 1- $\mu \mathrm{m}$ thick films to make the internal stress low [17].

\subsection{4}

\section{Specimen Fabrication}

The RRT specimen fabrication process was based on an on-chip tensile testing device. The process involves four lithography and etching steps: specimen shape definition, gauge mark fabrication, silicon wafer etching from the front to define the torsion bar thickness of the on-chip tensile testing device and wafer etching from the back side to release the specimens. The order of these steps differed according to the materials being tested.

The fabrication process for both SCS and polysilicon specimens was similar to the original process, with some steps added for gauge mark fabrication and pas- 
sivation of the test material and the gauge marks. We used back-side polished SOI and silicon wafers for the single-crystal silicon and polysilicon specimen processes, respectively. For SOI wafers, 1- $\mu$ m thick $\mathrm{SiO}_{2}$ film was deposited by plasma-enhanced chemical vapor deposition (PECVD) to passivate the top silicon layer. Then, all wafers were thermally oxidized $(0.5 \mu \mathrm{m})$ to create a sacrificial layer of the polysilicon specimens and the mask material for anisotropic etching from the back side. Then, LPCVD amorphous silicon film was deposited $(2 \mu \mathrm{m})$ and annealed in $\mathrm{N}_{2}$ at $1000^{\circ} \mathrm{C}$ to achieve crystallization. This "crystallized" polysilicon film was used as the back-side passivation film and also as the test material. After both single-crystal silicon and polysilicon films had been patterned to the specimen shape with reactive ion etching (RIE), titanium and titanium nitride film was deposited and patterned for the gauge marks. The silicon wafer was anisotropically etched using a tetramethylammonium hydroxide (TMAH) solution from both the front and back sides of the wafer. PECVD $\mathrm{SiO}_{2}$ films were used to passivate the specimen and gauge mark films. Finally, all oxide layers including sacrificial oxide were removed with buffered hydrofluoric acid (BHF) solution.

Anisotropic silicon etching for nickel specimen fabrication was done first from the front side of the thermally oxidized silicon wafer using potassium hydroxide $(\mathrm{KOH})$ solution. Then a 2- $\mu \mathrm{m}$ thick nickel film was electroplated using sputtered chromium and gold film as a seed layer. The thin gold film was deposited with sputtering and a lift-off process to produce the gauge marks. Then, anisotropic silicon etching from the back side was done. Finally, the thermal oxide film was removed with BHF.

Aluminum films were used in titanium specimen fabrication, as the sacrificial layer and passivation films for TMAH etching, instead of silicon dioxide film, because titanium film is attacked and damaged by the hydrofluoric acid used in silicon dioxide etching. The titanium film was deposited on the aluminum film and a thin gold film for the gauge marks was deposited and patterned. After the titanium film had been patterned to the specimen shape, the aluminum films were deposited again to passivate the specimen. Then, the silicon wafer was anisotropically etched from the back side of the wafer to release the specimen. The etchant was a silicon and ammonium persulfate-dissolved TMAH solution that did not attack the aluminum films [18].

Figure 1.6 shows a processed wafer of a single-crystal-silicon specimen before separating into chips, the three types of single-crystal silicon specimens and the type $\mathrm{C}$ specimens for gluing and electrostatic grip of each material.

\subsection{5}

\section{Results}

The results for each material except nickel were obtained from specimens on a single wafer. Nickel specimens were obtained from two wafers. The numbers of specimens tested for each material with each method are different, ranging from 1 to 13 . 


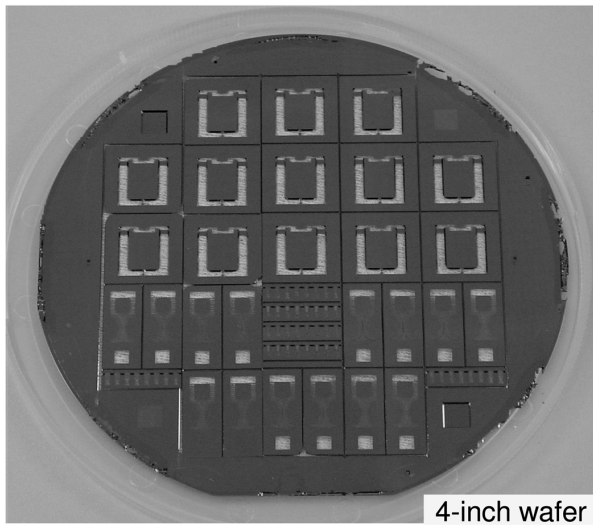

(a)

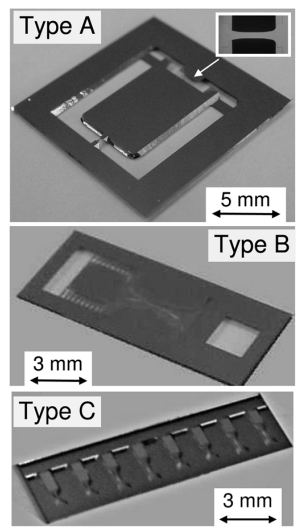

(b)
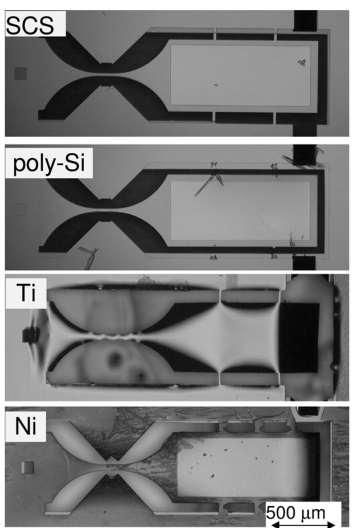

(c)

Figure 1.6 Fabricated round robin test specimens. (a) 4-inch silicon wafer of single-crystal silicon specimen, just finished the removal of the buried oxide, before cleaving into each chip; (b) three types of the single-crystal silicon specimen chips; (c) type $\mathrm{C}$ chip of each material.

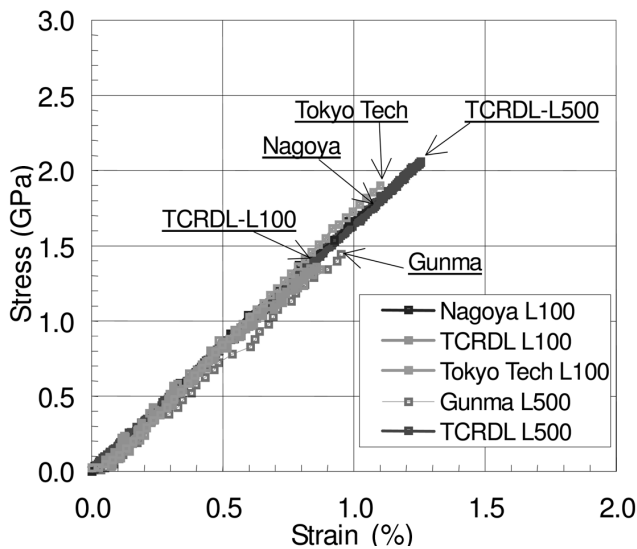

Figure 1.7 Stress-strain curves of single-crystal silicon film fabricated from SDB-SOI wafer. $(w=20 \mu \mathrm{m})$.

\subsubsection{Single-crystal Silicon and Polysilicon}

Both single-crystal silicon (SCS) films exhibited linear stress-strain relationships and there was good agreement among all the curves obtained by each method, as Figure 1.7 shows for the stress-strain curves of SCS/SDB obtained from each tensile test method. There was little difference in the stress-strain curves for polysilicon and some curves had a non-linear area in the high stress/strain region. The reason for this non-linear part is not clear, but we think that some specimen chucking problems occurred rather than plastic deformation. 


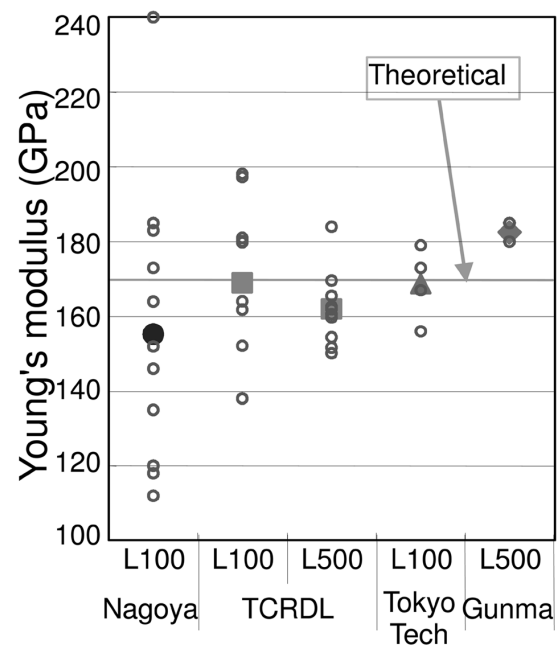

Figure 1.8 Young's modulus of single-crystal silicon from Epi-SOI.

The mechanical properties measured from the stress-strain curves showed good agreement among the test methods. The averages for the Young's modulus of SCS/SDB and SCS/Epi tested by each test method ranged from 135 to 219 and from 155 to $183 \mathrm{GPa}$, respectively. The averages for polysilicon ranged from 134 to $173 \mathrm{GPa}$. They ranged around the theoretical modulus. The theoretical Young's modulus of SCS specimens whose tensile axis was in the 110 direction was 168.9 GPa. The polysilicon film was (111) oriented. Young's modulus in the plane direction of (111) oriented polysilicon film did not depend on the in-plane orientation and was $168.9 \mathrm{GPa}$. The averages of three silicon films measured by TCRDL and Tokyo Tech ranged from 163 to $180 \mathrm{GPa}$, which well agreed with the theoretical values. The standard deviation for Young's modulus was about $10 \%$ of the average Young's modulus. The measured values and averages of Young's modulus of SCS/Epi are plotted in Figure 1.8.

The average tensile strength of SCS/SDB, SCS/Epi and polysilicon ranged from 1.49 to 2.05 , from 1.87 to 2.25 and from 1.44 to $2.51 \mathrm{GPa}$, respectively, and the average fracture strain ranged from 0.92 to 1.20 , from 0.87 to 1.45 and 0.96 to $1.52 \%$, which were largest in polysilicon, SCS/Epi and SCS/SDB in that order. This means that polysilicon had the smallest fracture origin. The Weibull plot of each silicon film is shown in Figure 1.9. The Weibull moduli of SCS/SDB, SCS/ Epi and polysilicon ranged from 4.7 to 8.6 , from 3.1 to 4.8 and from 7.3 to 16.5 , respectively, which showed that the deviation in strength of polysilicon was smaller than that in SCS. We can conclude that the deviation in defect size was uniform in the polysilicon specimen. The fracture origin of the silicon specimens was often located on RIE etched surfaces [9]. The etched surfaces of these materials may have had different roughnesses. 

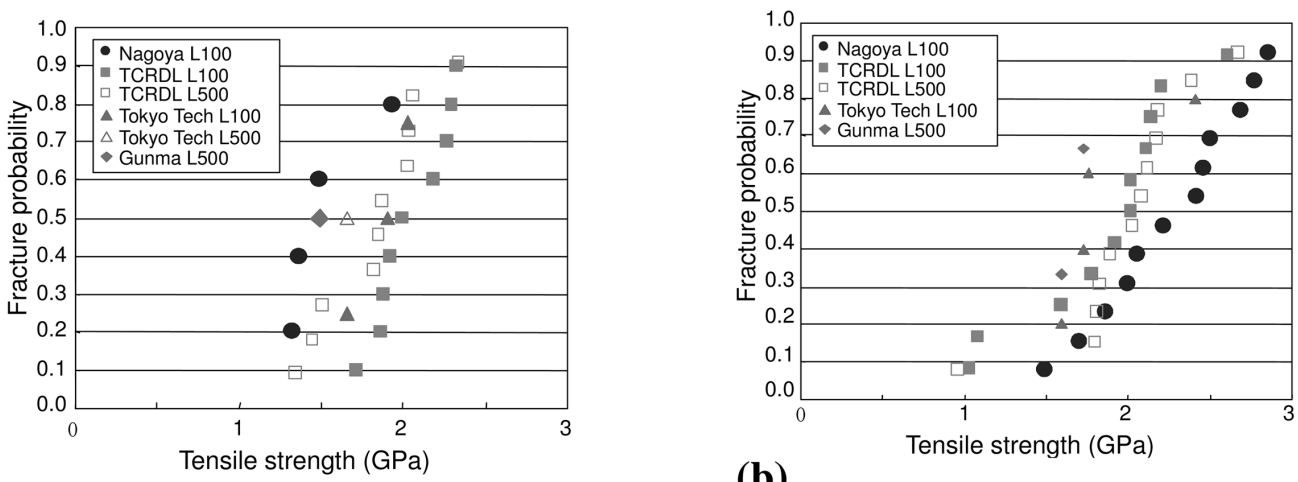

(a)

(b)

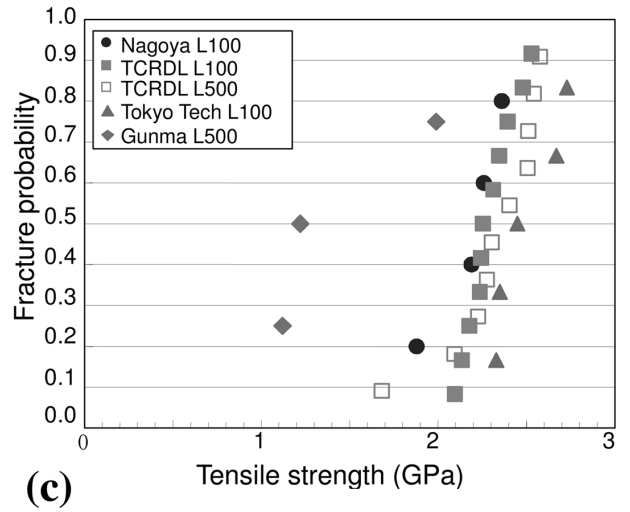

Figure 1.9 Weibull plots for silicon films. (a) Single-crystal silicon from SDB-SOI; (b) single-crystal silicon from Epi-SOI; (c) polysilicon.

\subsubsection{Nickel}

The nickel specimens exhibited brittle fractures with small plastic deformation after their yield point was identified. Fracture surfaces were parallel to the maximum shear stress directions. As shown in Figure 1.10, the stress-strain curves indicated a large difference in both the slope of the curves and the fracture strains between the specimens. The difference in the slopes reflects the difference in Young's modulus. The averages of the Young's modulus, tensile strength and fracture strain ranged from 49 to $185 \mathrm{GPa}$, from 0.54 to $2.18 \mathrm{GPa}$ and from 0.93 to $2.31 \%$, which showed much larger deviations than those of silicon films. Figure 1.11 shows the measured values and averages of Young's modulus and tensile strength. The largest maximum strains appeared between specimens tested using the mechanical grip and micro-gluing methods, where the loading rate was low. We have to equalize the loading rate in order to compare ductile materials. 


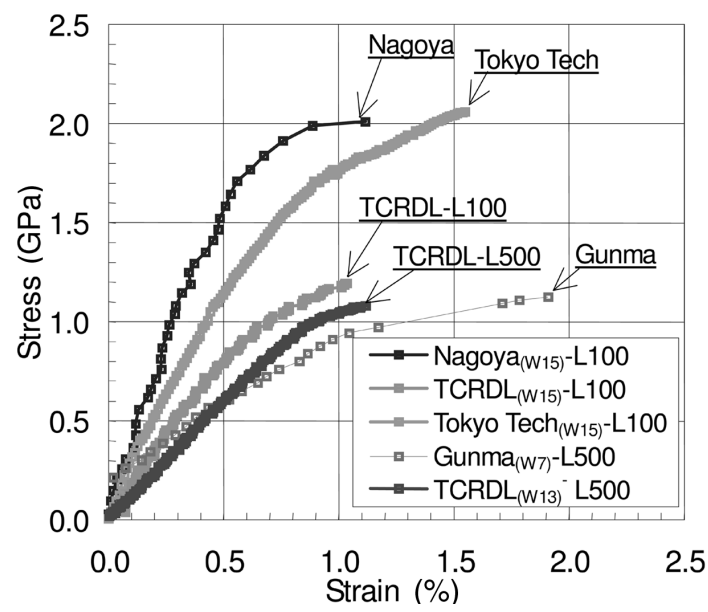

Figure 1.10 Stress-strain curves of electroplated nickel film.

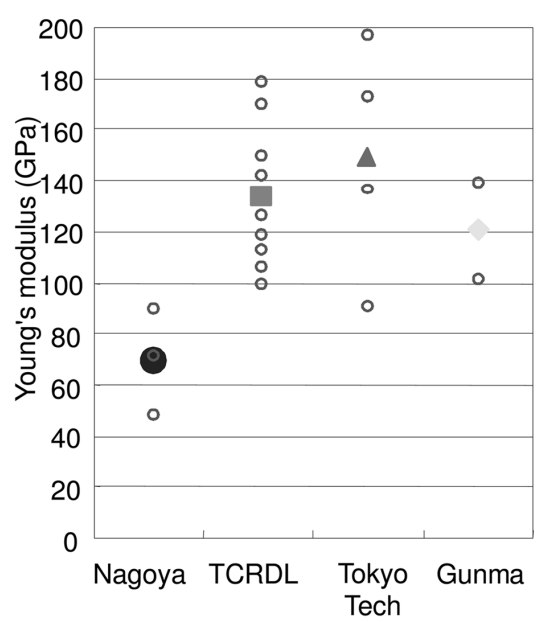

(a)

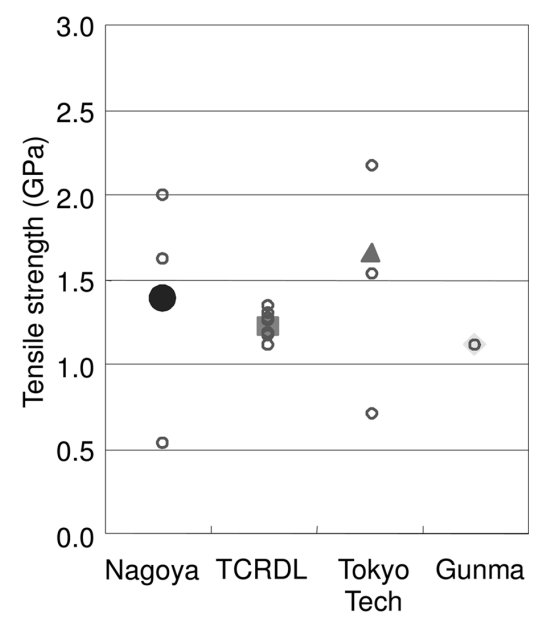

(b)

Figure 1.11 Mechanical properties of electroplated nickel film.

(a) Young's modulus; (b) tensile strength.

\subsubsection{Titanium}

The titanium specimens exhibited brittle fractures with plastic deformation after their yield point was identified as shown in the stress-strain curves in Figure 1.12. In contrast to the nickel films, there are small differences between test methods. The Young's modulus and tensile strength are plotted in Figure 1.13. The average Young's modulus was about $100 \mathrm{GPa}$, which is smaller than that of bulk titanium of 115 GPa [19]. The deviation in modulus was caused by the deviations in dimen- 


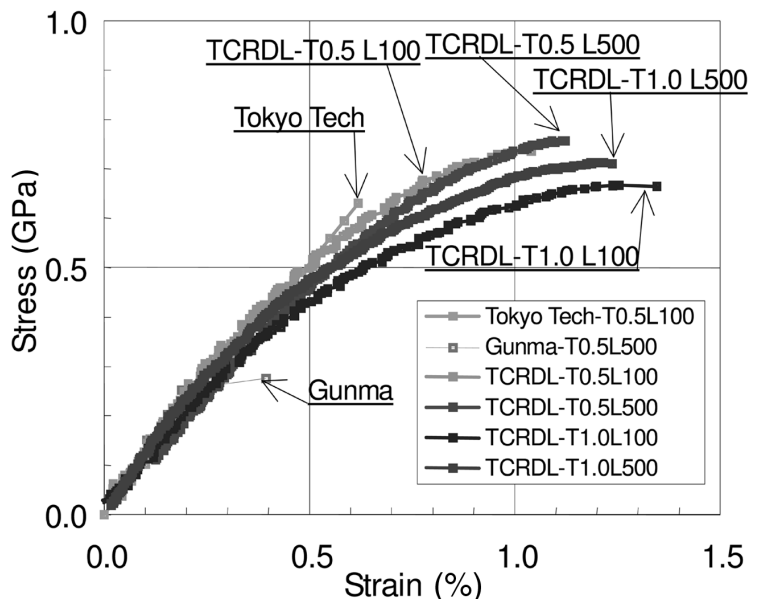

Figure 1.12 Stress-strain curves of sputtered titanium film.

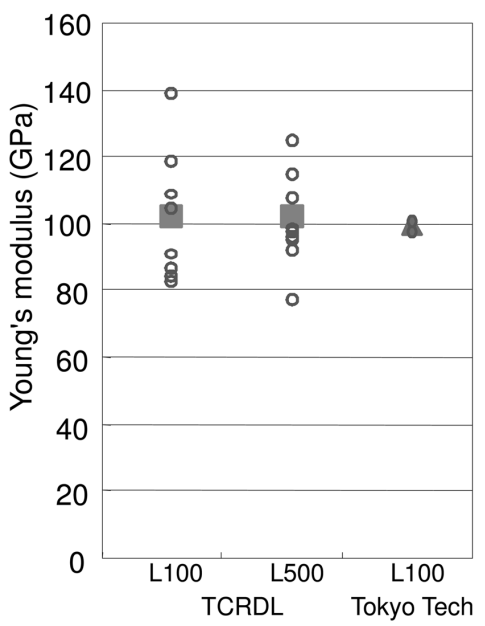

(a)

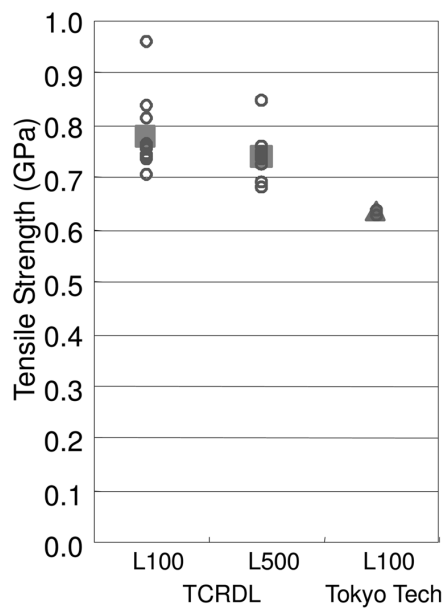

(b)

Figure 1.13 Mechanical properties of sputtered titanium film.

(a) Young's modulus; (b) tensile strength.

sions, especially specimen thickness. The average tensile strength ranged from 0.64 to $0.78 \mathrm{GPa}$. The deviation in strength was small. Some specimens had very large $(>10 \%)$ maximum strains. In these specimens, large slips along the maximum shear stress directions appeared. However, Ogawa et al. reported brittle fractures and small maximum elongations in tensile tests of sputtered titanium films [20]. 
This difference in fracture behavior may be caused by the deposition conditions [17].

Not all of the stress-strain curves of each test method for titanium films could be obtained because there were some problems, e.g. the specimens were too thin to calculate the applied load for on-chip tensile test methods or the specimens were damaged during the specimen chucking procedures used in mechanical grip methods.

\subsection{6}

\section{Discussion}

The RRT results revealed that there were no apparent differences between measuring methods and the measured properties and their deviations had almost the same values. Figure 1.9 shows the Weibull plots of the silicon specimens. The plotted points in each graph represent the strength of the specimen from one wafer. The slope of each plot is similar for the same materials. This means that the deviation in strength, i.e. the deviation in the size of the fracture origin, is the same, which means that specimens tested with all tensile test methods fractured in the same fracture mode. These results confirm the accuracy and repeatability of all these methods.

The standard deviation of the Young's modulus of silicon films ranged from 5 to $20 \%$, which was larger than the estimated deviation in specimen dimensions. We have to identify the source of these deviations in order to reduce them.

To compare the measured Young's modulus from each test method statistically, the hypothesis that "there is no difference between the two test methods in measuring Young's modulus" was tested using a $t$-test at significance level of $5 \%$. The hypothesis was accepted in most of the comparisons, but rejected in comparison with the on-chip tensile test method with the other methods. There are two possible reasons for the difference with the on-chip tensile test method. First, the mechanism that converts pushing force to tensile force would lead to errors in the force measurement. The rotation axis of the torsion bar would change during the testing. Second, the differential measurement would cause errors, because the difference in the Young's modulus was larger when the cross-section of the specimen was smaller. In such a case, the torsion bar stiffness is much larger than the specimen stiffness.

The mechanical grip methods had smaller values for strength measurement, which may have been caused by the effect of the size or stress concentration on the rounded part of the specimens that had a larger radius of curvature.

In summary, there were no systematic differences between the test techniques compared in this experiment. One can choose any method in the evaluation of the strength of thin films for his object, process compatibility or equipment. However, there is a weakness in each method. For example, the electrostatic grip has a limit of the maximum tensile force that can be applied to the specimen because of the gripping force limit. The on-chip tensile testing device has a limit of the maximum 
specimen length. The displacement applicable at the end of the specimen is limited by the torsion bar strength.

\section{5}

\section{International Standards on MEMS Materials}

The measurement of thin-film mechanical properties is crucial for the design and evaluation of MEMS devices. A lot of research has been carried out to evaluate the repeatability, accuracy and data reliability of various measurement methods for thin-film mechanical properties, because the information from these studies so far is not sufficient. The development of international standards on MEMS materials and their properties measurement methods will solve these problems. There had been an understanding that the MEMS industry does not require standards because of the variations in fabrication methods and dimensions. Recently, the development of standards in the MEMS field has been started in order to establish the fundamentals of reliability evaluations, especially on material properties. In this section, recent activities in the development of standards in MEMS are described. In addition, published international standards and currently active projects on measurements of mechanical properties are introduced.

\subsection{1}

\section{MEMS Standardization Activities}

\subsubsection{IEC}

The International Electrotechnical Commission (IEC) is the international standardization association for the electrical and electronics industries. The Technical Committee on Semiconductor Devices (TC47) has a working group on microelectromechanical systems (TC47/WG4). This working group is the only committee in the MEMS field in the international standardizing association. The group has published three international standards and is discussing three projects for new proposals on MEMS standards. The first published standard is on terminology (IEC62047-1), published in 2005. It contains more than 100 terms with definitions. The second and third one were published in 2006 on tensile test methods for thin films. Currently under discussion are standards on general definitions, RF-MEMS switches and fatigue test methods. Japan and Korea are active in the working group and each has a plan to propose new standard items in the future.

\subsubsection{ASTM International}

ASTM International is an international standards organization that develops and produces technical standards mainly for materials. The Technical Committee on Fatigue and Fracture (E08) has a Task Group (E08.05.03) on Structural Films and Electronic Materials that develops standards for electronic and micromechanical applications. Standards on in-plane length, residual strain and strain gradient measurement of thin films using an optical interferometer (E2244-06, E2245-06 
and E2246-06) have been published. They also carried out a round robin test on thin-film tensile testing [12].

\subsubsection{SEMI}

SEMI is an industry association mainly for semiconductor manufacturing supply. It is also working on standards related to semiconductor fabrication, such as the specification of wafers and process gases, an evaluation method for process techniques and process management. SEMI has an interest in MEMS devices due to the compatibility of their fabrication processes. Standardization in the MEMS fabrication area is an active consideration. Three standards have been published: Guide to Specifying Wafer-Wafer Bonding Alignment Targets (MS1-0307), Test Method for Step-height Measurements of Thin, Reflecting Films Using an Optical Interferometer (MS2-0307) and Terminology for MEMS Technology (MS3-0307). Development work on wafer bond strength test methods is in progress.

\subsubsection{Micromachine Center in Japan}

The Micromachine Center (MMC) is a nonprofit foundation dedicated to supporting the establishment of a technological basis of future micromachines/MEMS, and to support the development of MEMS/micromachine industries. MMC is dedicated to standardization in this field and has published technical reports on terminology and evaluation methods for material properties. Based on these studied and projects on standardization research supported by the government, they have proposed standards on terminology, tensile test methods and fatigue test methods to IEC TC47/WG4.

\subsection{2}

\section{International Standards on Thin-film Uniaxial Stress Testing}

The IEC standard on tensile test methods for thin-film materials (IEC 62047-2) specifies tensile test methods for thin-film materials whose thickness is less than $10 \mu \mathrm{m}$ and lateral dimensions (length and width) are less than $1 \mathrm{~mm}$. This standard is a guide for the repeatable, reliable and accurate tensile testing of thin films, in which the specifications of specimen, test machine and test conditions are identified, but a specific test method is not required. In the Section 4 on test methods and test apparatus, specimen gripping is pointed out as an important specification for test apparatus. Some gripping methods are explained in an appendix. In Section 5 on specimens, the specification of the specimen is described, which considers dimension errors and shape errors of microfabricated specimens.

The IEC standard on thin-film standard test pieces for tensile testing (IEC 620473) specifies a standard test piece for thin-film tensile testing in order to qualify the accuracy and repeatability of a tensile testing machine. This standard recommends using single-crystal silicon as a standard specimen.

These two standards related to tensile testing of thin films were compiled from the results of cross-comparisons among tensile test methods described in the previous section. Currently, a new standard on axial fatigue test methods for thin- 
film materials is being discussed, which is based on the international standard on fatigue test methods for metallic materials (ISO 1099) and referred to IEC 62047-2. The differences between bulk and thin films are specified.

\section{6 \\ Conclusion}

In this chapter, the contribution of the mechanical properties of thin films to the properties of MEMS devices, especially reliability, has been described. Not only the strength and fatigue, but also the elastic properties are important for MEMS reliability. There are many difficulties in their evaluation, which mainly come from their small dimensions. The cross-comparison of evaluation methods to solve these difficulties and international standards compiled from the results of such comparisons are described.

In order to realize highly functional and highly reliable MEMS devices, accurate, repeatable properties should be measured, in which the measured properties can be compared with each other. These activities should extend to various evaluations in MEMS research and development.

\section{References}

1 L. Hornbeck, Digital light processing and MEMS: timely convergence for a bright future, Proc. SPIE 2639 (1995) 2.

2 K. Sato, T. Yoshioka, T. Ando, M. Shikida, T. Kawabata, Tensile testing of silicon film having different crystallographic orientations carried out on a silicon chip, Sens. Actuators A 70 (1998) 148-152.

3 T. Yi, L. Li, C. J. Kim, Microscale material testing of single crystalline silicon: process effects on surface morphology and tensile strength, Sens. Actuators A 83 (2000) 172-178.

4 J. Å. Schweitz, F. Ericson, Evaluation of mechanical materials properties by means of surface micromachined structure, Sens. Actuators A 74 (1999) 126-133.

5 W. N. Sharpe, B. Yuan, R. Vaidyanathan, R. L. Edwards, New test structures and techniques for measurement of MEMS Materials, Proc. SPIE 2880 (1996) 78-91.

6 W. N. Sharpe, K. T. Turner, R. L. Edwards, Tensile testing of polysilicon, Exp. Mech. 39 (1999) 162-170.
7 J. Koskinen, J. E. Steinwall, R. Soave, H. H. Johnson, Microtensile testing of free-standing polysilicon fibers of various grain sizes, J. Micromech. Microeng. 3 (1993) 13-17.

8 S. Greek, F. Ericson, S. Johansson, M. Fürtsch, A. Rump, Mechanical characterization of thick polysilicon films: Young's modulus and fracture strength evaluated with microstructures, $J$. Micromech. Microeng. 9 (1999) 245-251.

9 T. Tsuchiya, O. Tabata, J. Sakata, Y. Taga, Specimen size effect on tensile strength of surface micromachined polycrystalline silicon thin films, J. Micro ElectroMech. Syst. 7 (1998) 106-113.

10 H. Kapels, R. Aigner, J. Binder, Fracture strength and fatigue of polysilicon determined by a novel thermal actuator, IEEE Trans. Electron Devices, 47 (2000) 1522-1528.

11 I. Chasiotis, W. G. Knauss, Microtensile tests with the aid of probe microscopy for the study of MEMS materials, Proc. SPIE 4175 (2000) 92-102.

12 D. A. LaVan, T. Tsuchiya, G. Coles, W. G. Knauss, I. Chasotis, D. Read, Cross 
comparison of direct strength testing techniques on polysilicon films, in Mechanical Properties of Structural Films, ASTM STP 1413, ASTM, Philadelphia, 16-26, 2001.

13 Y. Saotome, Y. Nakazawa, S. Kinuta, Microfactory cells for in-situ micromaterials and microstructures testing, in Proceedings of the 2nd International Workshop on Microfact., Fribourg, Switzerland, October 9-10, 2000, 99-102, 2000.

14 Y. Higo, K. Takashima, M. Shimojo, S. Sugiura, B. Pfister, M. V. Swain, Fatigue testing machine of micro-sized specimens for MEMS applications, Mater. Res. Soc. Symp. Proc. 605 (2000) 241-246.

15 T. Yonehara, K. Sakaguchi, ELTRAN ${ }^{\circledR}$; novel SOI wafer technology, JSAP Int. 4 (2001) 11-16.

16 H. Funabashi, T. Tsuchiya, Y. Kageyama, J. Sakata, Fabrication technology of three layer polysilicon microstructures without CMP for gyroscope, in Technical Digest of the 10th International Conference of SolidState Sensors and Actuators (Transducers 99), Sendai, Japan, June 7-10, 1999, 336-339, 1999.

17 T. Tsuchiya, M. Hirata, N. Chiba, Young's modulus, fracture strain and tensile strength of sputtered titanium thin films, Thin Solid Films 484 (2005) 245-250.

18 G. Yan, P. C. H. Chan, I.-M. Hsing, R. K. Sharma, J. K. O. Sin, Y. Wang, An improved TMAH Si-etching solution without attacking exposed aluminum, Sens. Actuators A 89 (2001) 135-141.

19 M. Chinmulgund, R. B. Inturi, J. A. Barnard, Effect of Ar gas pressure on growth, structure and mechanical properties of sputtered $\mathrm{Ti}, \mathrm{Al}, \mathrm{TiAl}$ and Ti3Al films, Thin Solid Films 270 (1995) 260-263.

20 H. Ogawa, K. Suzuki, S. Kaneko, Y. Nakano, Y. Ishikawa, T. Kitahara, Tensile testing of microfabricated thin films, Microsystem Technologies, 3 (1997) 117-121. 
